\title{
Misspecified Cramér-Rao Bounds for Complex Unconstrained and Constrained Parameters
}

\author{
Stefano Fortunati \\ Dipartimento di Ingegneria dell'Informazione, University of Pisa, 56122 Pisa, Italy \\ Email: stefano.fortunatidiet.unipi.it
}

\begin{abstract}
In this paper, a generalization of the Misspecified Cramér-Rao Bound (MCRB) and of the Constrained MCRB (CMCRB) to complex parameter vectors is presented. Our derivation aims at providing lower bounds on the Mean Square Error (MSE) for both circular and non-circular, $M S$-unbiased, mismatched estimators. A simple toy example is also presented to clarify the theoretical findings.
\end{abstract}

\section{INTRODUCTION}

Recent papers have discussed the possibility to establish a lower bound on the error covariance matrix of an estimator in the presence of model misspecification (see e.g. [1], [2], [3]). The model misspecification problem arises when the true data model (or the true data probability density function, pdf) is different from the one assumed to derive an estimator of the parameters of interest. A lower bound under misspecification, named Misspecified Cramér-Rao Bound (MCRB), has been firstly derived by Vuong in his seminal working paper [4] for real parameter vectors. More recently, a different proof of the same MCRB has been proposed by Richmond and Horowitz in [1], where an extension of this bound to complex parameter vectors has been also provided and applied to the DOA estimation problem. In [5], the MCRB for the joint DOA-DOD estimation in MIMO radars has been obtained by stacking the real and the imaginary parts of the complex parameters. In this paper, we provide a general expression of the MCRB for complex unconstrained (Theorem 1) and constrained (Theorem 2) parameter vectors that can be applied to both circular and non-circular (i.e. with a non-vanishing pseudo-covariance), $M S$-unbiased, mismatched estimators. Our strategy to derived the complex MCRB follows the one adopted in [6] and [7] for the complex unconstrained and constrained CRB. Specifically, we will not derive any new covariance inequality directly in the complex field (as done e.g. in [8] and [9] for the classical $\mathrm{CRB}$ ) but, relying on the interrelated representations of vectors in $\mathbb{C}^{N}, \mathbb{C}^{2 N}$ and $\mathbb{R}^{2 N}$, we will transform the real MCRB in its complex form. Although less general than the framework detailed in [9], our approach avoids technical complications implied by reproducing the proofs given in [4] and [3] for real parameters directly on the complex field.

On account of the limited space available, we assume that the reader is already aware of the main findings about the misspecified estimation framework detailed in [1], [2], [3], [4] and in the references therein. Moreover, we also assume that the reader is familiar with the basic notions on complex random variables, complex pdfs, complex expectations ( [10],
[11], [12], [13], [14], [15]) and with the so-called Wirtinger or $\mathbb{C R}$-calculus ( [16], [17], [18], [19], [20] and the references therein).

Notation: Throughout this paper, italics indicates scalar quantities $(a)$, lower case and upper case boldface indicate column vectors (a) and matrices (A) respectively. $*$ indicates the complex conjugation. The superscripts $T$ and $H$ indicates the transpose and the Hermitian operators, then $\mathbf{A}^{H}=\left(\mathbf{A}^{*}\right)^{T}$. For two matrices $\mathbf{A}$ and $\mathbf{B}, \mathbf{A} \geq \mathbf{B}$ means that $\mathbf{A}-\mathbf{B}$ is positive semi-definite.

\section{Preliminaries}

In order to make this paper as self-contained as possible, in this section, we provide a brief summary of the main definitions, notations and operators that will play a fundamental role in the derivation of the MCRB for complex parameter vectors. Our main references here are the book [16] and the excellent tutorial paper [17]. We focus our attention on the class of scalar real valued functions of complex variables. In particular, let $\mathbf{v}=\mathbf{x}+j \mathbf{y} \in \mathbb{C}^{N}$ be a complex vector and let $f: \mathbb{C}^{N} \rightarrow \mathbb{R}$ be a real valued function. The class of real valued functions is extensively used in signal processing applications. For example, the likelihood functions or the error loss functions are members of this class. Except for the constant function, a real valued function is not holomorphic, and consequently the classical complex functional analysis cannot be exploited ( [16], [17]). A way to overcome this limitation is to represent a real valued non-holomorphic function $f(\mathbf{v})$ as function of the real and the imaginary parts of $\mathbf{v}$, i.e. $f(\mathbf{x}, \mathbf{y})$ or alternatively as function of both $\mathbf{v}$ and its complex conjugate $\mathbf{v}^{*}$, i.e. $f\left(\mathbf{v}, \mathbf{v}^{*}\right)$. To this end, we will make extensive use of the following two mappings ( [6], [9], [16] and [17]). We define as the real representation of $\mathbf{v}$ the mapping:

$$
\begin{aligned}
\sim & : \mathbb{C}^{N} \rightarrow \mathbb{R}^{2 N} \\
& \mathbf{v} \mapsto \tilde{\mathbf{v}}=\left(\mathbf{x}^{T}, \mathbf{y}^{T}\right)^{T},
\end{aligned}
$$

and as complex augmented representation of $\mathbf{v}$ the mapping:

$$
\begin{aligned}
\therefore: & \mathbb{C}^{N} \rightarrow \mathcal{C} \subset \mathbb{C}^{2 N} \\
\quad \mathbf{v} & \mapsto \underline{\mathbf{v}}=\left(\mathbf{v}^{T}, \mathbf{v}^{H}\right)^{T} .
\end{aligned}
$$

The mapping (1) represents the natural set isomorphism between $\mathbb{C}^{N}$ and $\mathbb{R}^{2 N}$ while (2) defines an invertible mapping between $\mathbb{C}^{N}$ and $\mathcal{C} \subset \mathbb{C}^{2 N}$. It is worth noticing that $\mathcal{C}$ is not a linear subspace of $\mathbb{C}^{2 N}$. Indeed, $\mathcal{C}$ can be viewed as vector 
space of dimension $2 N$ on the real field [17]. In particular, there exists a linear and invertible transformation $T$ such that:

$$
\begin{aligned}
T & : \mathbb{R}^{2 N} \rightarrow \mathcal{C} \subset \mathbb{C}^{2 N} \\
\tilde{\mathbf{v}} & \mapsto \underline{\mathbf{v}}=T(\tilde{\mathbf{v}}) \triangleq \mathbf{T}_{2 N} \tilde{\mathbf{v}}
\end{aligned}
$$

where $\mathbf{T}_{2 N}$ is explicitly defined as:

$$
\underline{\mathbf{v}}=\mathbf{T}_{2 N} \tilde{\mathbf{v}}=\left(\begin{array}{cc}
\mathbf{I}_{N} & j \mathbf{I}_{N} \\
\mathbf{I}_{N} & -j \mathbf{I}_{N}
\end{array}\right)\left(\begin{array}{l}
\mathbf{x} \\
\mathbf{y}
\end{array}\right)
$$

where $\mathbf{I}_{N}$ is the identity matrix of dimension $N$ and $\mathbf{T}_{2 N}$ is a unitary matrix up to a scalar factor of 2 , i.e. $\mathbf{T}_{2 N}^{H}=2 \mathbf{T}_{2 N}^{-1}$. Unless otherwise stated, the size of $\mathbf{T}$ is $2 N$, so the related subscript will be dropped for notation simplicity. We refer to [10] and [17] for a complete list of properties of $\mathbf{T}$. Using the relations (1), (2) and (3), with an abuse of notation, a real valued function $f(\mathbf{v})$ will be interchangeably represented as $f(\mathbf{x}, \mathbf{y}), f(\tilde{\mathbf{v}}), f\left(\mathbf{v}, \mathbf{v}^{*}\right)$ or $f(\underline{\mathbf{v}})$. Actually, using the relation in (1), a real valued functions can be always represented as $f: \mathbb{R}^{2 N} \rightarrow \mathbb{R}$, then the classical real analysis could be applied straight. However, to work directly within the complex variables framework will preserve a more clear insight about the problem at hand and will guarantee a simpler algebraic manipulations of the equations. The rest of this section is devoted to define the complex gradient and the complex Hessian matrix of $f$. We firstly introduce the two row operators, i.e. the Wirtinger derivatives, as:

$$
\begin{aligned}
& \frac{\partial f\left(\mathbf{v}, \mathbf{v}^{*}\right)}{\partial \mathbf{v}}=\frac{1}{2}\left(\frac{\partial f(\mathbf{x}, \mathbf{y})}{\partial \mathbf{x}}-j \frac{\partial f(\mathbf{x}, \mathbf{y})}{\partial \mathbf{y}}\right) \\
& \frac{\partial f\left(\mathbf{v}, \mathbf{v}^{*}\right)}{\partial \mathbf{v}^{*}}=\frac{1}{2}\left(\frac{\partial f(\mathbf{x}, \mathbf{y})}{\partial \mathbf{x}}+j \frac{\partial f(\mathbf{x}, \mathbf{y})}{\partial \mathbf{y}}\right)
\end{aligned}
$$

where $\mathbf{v}$ and $\mathbf{v}^{*}$ have to be formally considered as two different variables. In [17], these operators are called cogradient operator and conjugate cogradient operator, respectively. Moreover, in [17] it is shown that the gradient, i.e. the vector in the tangent space defining the direction of the maximum rate of change of the function $f$, is given by:

$$
\nabla_{\mathbf{v}} f \triangleq\left(\frac{\partial f\left(\mathbf{v}, \mathbf{v}^{*}\right)}{\partial \mathbf{v}}\right)^{H}=\left(\frac{\partial f\left(\mathbf{v}, \mathbf{v}^{*}\right)}{\partial \mathbf{v}^{*}}\right)^{T}
$$

where the second equality follows from the assumption that $f$ is a real valued function. As for the standard real multivariate case, a necessary and sufficient condition for the real valued function $f(\mathbf{v})$ to have a stationary point in $\mathbf{v}_{0}$ is that the gradient in (7) vanishes at $\mathbf{v}_{0}$, i.e. $\nabla_{\mathbf{v}_{0}} f=\mathbf{0}$. Before introducing the Hessian matrix, we define the row differential operators:

$$
\begin{gathered}
\partial / \partial \tilde{\mathbf{v}} \triangleq\left(\begin{array}{cc}
\partial / \partial \mathbf{x} \quad \partial / \partial \mathbf{y}
\end{array}\right)=\left(\begin{array}{cc}
\nabla_{\mathbf{x}}^{T} & \nabla_{\mathbf{y}}^{T}
\end{array}\right) \triangleq \nabla_{\tilde{\mathbf{v}}}^{T} f \\
\partial / \partial \underline{\mathbf{v}} \triangleq\left(\begin{array}{cc}
\partial / \partial \mathbf{v} & \partial / \partial \mathbf{v}^{*}
\end{array}\right)
\end{gathered}
$$

named real cogradient and complex augmented cogradient, respectively. The operator in (8) acts on $f$, taken as a real function of real variables, as the classical (real) cogradient operator. As detailed in [17], the real cogradient in (8) can be transformed in the complex augmented cogradient in (9) and vice versa, by means of the invertible transformation $\mathbf{T}$ in (3):

$$
\nabla_{\tilde{\mathbf{v}}}^{T} f=\frac{\partial f(\tilde{\mathbf{v}})}{\partial \tilde{\mathbf{v}}}=\frac{\partial f(\underline{\mathbf{v}})}{\partial \underline{\mathbf{v}}} \mathbf{T} .
$$

At this point, we can introduce the (real) Hessian matrix of $f$ in the classical real sense as:

$$
\mathbf{H}_{\tilde{\mathbf{v}} \tilde{\mathbf{v}}}=\frac{\partial}{\partial \tilde{\mathbf{v}}}\left(\frac{\partial f(\tilde{\mathbf{v}})}{\partial \tilde{\mathbf{v}}}\right)^{T}=\frac{\partial}{\partial \tilde{\mathbf{v}}}\left(\frac{\partial f(\tilde{\mathbf{v}})}{\partial \tilde{\mathbf{v}}}\right)^{H}=\nabla_{\tilde{\mathbf{v}}}^{T} \nabla_{\tilde{\mathbf{v}}} f
$$

It is immediate to verify that a complex representation of the Hessian matrix can be obtained by applying the transformation in (10) as [17]:

$$
\mathbf{H}_{\tilde{\mathbf{v}} \tilde{\mathbf{v}}}=\mathbf{T}^{H} \mathbf{H}_{\underline{\mathbf{v v}}} \mathbf{T}=\mathbf{T}^{H}\left(\begin{array}{cc}
\mathbf{H}_{\mathbf{v v}} & \mathbf{H}_{\mathbf{v}^{*} \mathbf{v}} \\
\mathbf{H}_{\mathbf{v} \mathbf{v}^{*}} & \mathbf{H}_{\mathbf{v}^{*} \mathbf{v}^{*}}
\end{array}\right) \mathbf{T},
$$

where

$$
\begin{aligned}
& \mathbf{H}_{\mathbf{v} \mathbf{v}}=\frac{\partial}{\partial \mathbf{v}}\left(\frac{\partial f\left(\mathbf{v}, \mathbf{v}^{*}\right)}{\partial \mathbf{v}}\right)^{H}=\nabla_{\mathbf{v}}^{H} \nabla_{\mathbf{v}} f=\mathbf{H}_{\mathbf{v} \mathbf{v}}^{H} \\
& \mathbf{H}_{\mathbf{v}^{*} \mathbf{v}}=\frac{\partial}{\partial \mathbf{v}^{*}}\left(\frac{\partial f(\mathbf{v})}{\partial \mathbf{v}}\right)^{H}=\nabla_{\mathbf{v}}^{T} \nabla_{\mathbf{v}} f=\mathbf{H}_{\mathbf{v} \mathbf{v}^{*}}^{*}
\end{aligned}
$$

and $\mathbf{H}_{\mathbf{v v}}^{H}=\mathbf{H}_{\mathbf{v}^{*} \mathbf{v}^{*}}^{*}$. Finally, it can be shown that the inverse transformation of eq. (12) is $\mathbf{H}_{\mathbf{v v}}=4^{-1} \mathbf{T} \mathbf{H}_{\tilde{\mathbf{v}} \tilde{\mathbf{v}}} \mathbf{T}^{H}$.

\section{THE COMPLEX UNCONSTRAINED MCRB}

Let $\mathbf{z}=\mathbf{x}+j \mathbf{y} \in \mathbb{C}^{N}$ be a complex random vector representing the outcome of a random experiment whose true but possibly unknown pdf is given by $p_{Z}(\mathbf{z})$. Following the classic misspecified framework, we assume that $\mathbf{z}$ is sampled from a pdf $f_{Z}(\mathbf{z} ; \boldsymbol{\theta})$ parameterized by a complex unknown deterministic parameter vector $\boldsymbol{\theta}=\boldsymbol{\alpha}+j \boldsymbol{\beta} \in \Theta \subseteq \mathbb{C}^{d}$. As usual, $f_{Z}(\mathbf{z} ; \boldsymbol{\theta})$ can possibly be different from $p_{Z}(\mathbf{z})$ for any $\boldsymbol{\theta} \in \Theta$. Moreover, let $\hat{\boldsymbol{\theta}} \triangleq \hat{\boldsymbol{\theta}}(\mathbf{z})=\hat{\boldsymbol{\alpha}}(\mathbf{z})+j \hat{\boldsymbol{\beta}}(\mathbf{z}) \in \mathbb{C}^{d}$ be the complex valued estimator of $\boldsymbol{\theta}$ derived under the misspecified pdf $f_{Z}(\mathbf{z} ; \boldsymbol{\theta})$. The strategy that we will follow to derive a complex MCRB is similar to the one adopted in [6] for the CRB. In particular, we exploit the mappings in (1), (2) and (3) to represent in a complex form the MCRB derived in the classical real framework, by keeping unchanged the main proof about the real MCRB.

In the rest of this section, we assume that:

Al Given the relation $(1), p_{Z}(\mathbf{z})$ and $f_{Z}(\mathbf{z} ; \boldsymbol{\theta})$ admit an alternative, yet equivalent, representation as $p_{Z}(\tilde{\mathbf{z}})$ and $f_{Z}(\tilde{\mathbf{z}} ; \tilde{\boldsymbol{\theta}})$.

A2 The pdfs $p_{Z}(\tilde{\mathbf{z}})$ and $f_{Z}(\tilde{\mathbf{z}} ; \tilde{\boldsymbol{\theta}})$ belong to two (generally different) families (or models) $\mathcal{P}$ and $\mathcal{F}$. Moreover, we assume that $\mathcal{F}$ is regular with respect to $\mathcal{P}$ (see [2], [4]).

A3 The function $E_{p}\left\{\ln f_{Z}(\tilde{\mathbf{z}} ; \tilde{\boldsymbol{\theta}}) \mid \tilde{\boldsymbol{\theta}} \in \tilde{\Theta} \subseteq \mathbb{R}^{2 d}\right\}$ has a unique maximum on $\tilde{\Theta}$ in an interior point $\tilde{\boldsymbol{\theta}}_{0}$. Moreover, the matrix

$$
\mathbf{A}_{\tilde{\boldsymbol{\theta}}_{0}} \triangleq E_{p}\left\{\nabla_{\tilde{\boldsymbol{\theta}}_{0}}^{T} \nabla_{\tilde{\boldsymbol{\theta}}_{0}} \ln f_{Z}\left(\tilde{\mathbf{z}} ; \tilde{\boldsymbol{\theta}}_{0}\right)\right\}
$$

is non-singular at $\tilde{\boldsymbol{\theta}}_{0}$. 
A4 $\hat{\boldsymbol{\theta}}$ is a misspecified (MS)-unbiased ( [2], [4]) estimator, i.e. $E_{p}\{\hat{\boldsymbol{\theta}}\}=\int \hat{\boldsymbol{\theta}}(\mathbf{z}) p_{Z}(\mathbf{z}) d \mathbf{z}=\boldsymbol{\theta}_{0}$, where the expectation operator is defined as in [13].

As discussed in [1], [2] and [4], $\tilde{\boldsymbol{\theta}}_{0}$ can be seen as the real representation of the pseudo-true parameter vector, i.e. the point that minimizes the Kullback-Leibler (KL) divergence between $p_{Z}(\mathbf{z})$ and $f_{Z}(\mathbf{z} ; \boldsymbol{\theta})$ :

$$
\boldsymbol{\theta}_{0} \triangleq \underset{\boldsymbol{\theta} \in \Theta}{\arg \min }\left\{D\left(p_{Z} \| f_{\boldsymbol{\theta}}\right)\right\}=\underset{\boldsymbol{\theta} \in \Theta}{\arg \min }\left\{D\left(p_{Z} \| f_{\boldsymbol{\theta}}\right)\right\},
$$

where $D\left(p_{Z} \| f_{\boldsymbol{\theta}}\right) \triangleq E_{p}\left\{\ln \left(p_{Z}(\mathbf{z}) / f_{Z}(\mathbf{z} ; \boldsymbol{\theta})\right)\right\}$.

Under Assumptions A1-A4, Theorem 4.1 in [4] (or Theorem 1 in [2]) can be recast as follow:

Lemma 1. Let $\tilde{\hat{\boldsymbol{\theta}}}$ be the real representation of an MS-unbiased estimator $\hat{\boldsymbol{\theta}}$ derived under the misspecified model $\mathcal{F}$ from the data vector $\mathbf{z}$. Then for every $p_{Z}(\tilde{\mathbf{z}})$ in $\mathcal{P}$ :

$$
\mathbf{C}\left(\tilde{\hat{\boldsymbol{\theta}}}, \tilde{\boldsymbol{\theta}}_{0}\right) \geqslant \mathbf{A}_{\tilde{\boldsymbol{\theta}}_{0}}^{-1} \mathbf{B}_{\tilde{\boldsymbol{\theta}}_{0}} \mathbf{A}_{\tilde{\boldsymbol{\theta}}_{0}}^{-1} \triangleq \operatorname{MCRB}\left(\tilde{\boldsymbol{\theta}}_{0}\right),
$$

where

$$
\mathbf{C}\left(\tilde{\hat{\boldsymbol{\theta}}}, \tilde{\boldsymbol{\theta}}_{0}\right) \triangleq E_{p}\left\{\left(\tilde{\hat{\boldsymbol{\theta}}}-\tilde{\boldsymbol{\theta}}_{0}\right)\left(\tilde{\hat{\boldsymbol{\theta}}}-\tilde{\boldsymbol{\theta}}_{0}\right)^{T}\right\},
$$

is the (real) error covariance matrix of $\tilde{\hat{\boldsymbol{\theta}}}, \mathbf{A}_{\tilde{\boldsymbol{\theta}}_{0}}$ is defined as in (15) and:

$$
\mathbf{B}_{\tilde{\boldsymbol{\theta}}_{0}} \triangleq E_{p}\left\{\nabla_{\tilde{\boldsymbol{\theta}}_{0}} \ln f_{Z}\left(\tilde{\mathbf{z}} ; \tilde{\boldsymbol{\theta}}_{0}\right) \nabla_{\tilde{\boldsymbol{\theta}}_{0}}^{T} \ln f_{Z}\left(\tilde{\mathbf{z}} ; \tilde{\boldsymbol{\theta}}_{0}\right)\right\}
$$

Proof: Since all the quantities are given in terms of real vectors and functions, the proof of Lemma 1 is exactly the same of the one established by Vuong for his Theorem 4.1 [4]. Now, we can show that, by using the mappings (1), (2) and (3), it is possible to establish the MCRB directly in the complex field.

Theorem 1. Let $\hat{\boldsymbol{\theta}}$ be the complex augmented representation of an MS-unbiased estimator $\hat{\boldsymbol{\theta}}$ derived under the misspecified model $\mathcal{F}$ from the data vector $\mathbf{z}$. Then for every $p_{Z}(\mathbf{z})$ in $\mathcal{P}$ :

$$
\mathbf{C}\left(\underline{\hat{\boldsymbol{\theta}}}, \underline{\boldsymbol{\theta}}_{0}\right) \geqslant \mathbf{A}_{\underline{\boldsymbol{\theta}}_{0}}^{-1} \mathbf{B}_{\underline{\boldsymbol{\theta}}_{0}} \mathbf{A}_{\underline{\boldsymbol{\theta}}_{0}}^{-1} \triangleq \operatorname{MCRB}\left(\underline{\boldsymbol{\theta}}_{0}\right)
$$

where

$$
\mathbf{C}\left(\underline{\hat{\theta}}, \underline{\boldsymbol{\theta}}_{0}\right) \triangleq\left(\begin{array}{cc}
\mathbf{C}_{\mathbf{C}}\left(\hat{\boldsymbol{\theta}}, \boldsymbol{\theta}_{0}\right) & \mathbf{C}_{\mathbf{P}}\left(\hat{\boldsymbol{\theta}}, \boldsymbol{\theta}_{0}\right) \\
\mathbf{C}_{\mathbf{P}}\left(\hat{\boldsymbol{\theta}}, \boldsymbol{\theta}_{0}\right)^{*} & \mathbf{C}_{\mathbf{C}}\left(\hat{\boldsymbol{\theta}}, \boldsymbol{\theta}_{0}\right)^{*}
\end{array}\right),
$$

is the error covariance matrix of $\underline{\hat{\theta}}$ and the block-matrices

$$
\begin{aligned}
& \mathbf{C}_{\mathbf{C}}\left(\hat{\boldsymbol{\theta}}, \boldsymbol{\theta}_{0}\right) \triangleq E_{p}\left\{\left(\hat{\boldsymbol{\theta}}-\boldsymbol{\theta}_{0}\right)\left(\hat{\boldsymbol{\theta}}-\boldsymbol{\theta}_{0}\right)^{H}\right\}, \\
& \mathbf{C}_{\mathbf{P}}\left(\hat{\boldsymbol{\theta}}, \boldsymbol{\theta}_{0}\right) \triangleq E_{p}\left\{\left(\hat{\boldsymbol{\theta}}-\boldsymbol{\theta}_{0}\right)\left(\hat{\boldsymbol{\theta}}-\boldsymbol{\theta}_{0}\right)^{T}\right\},
\end{aligned}
$$

are the error covariance and pseudo-covariance matrices of $\hat{\boldsymbol{\theta}}$. The matrices $\mathbf{A}_{\underline{\theta}_{0}}$ and $\mathbf{B}_{\underline{\theta}_{0}}$ can be expressed as:

$$
\mathbf{A}_{\underline{\boldsymbol{\theta}}_{0}} \triangleq\left(\begin{array}{cc}
\mathbf{A}_{\boldsymbol{\theta}_{0} \boldsymbol{\theta}_{0}} & \mathbf{A}_{\boldsymbol{\theta}_{0}^{*} \boldsymbol{\theta}_{0}} \\
\mathbf{A}_{\boldsymbol{\theta}_{0}^{*}}^{*} \boldsymbol{\theta}_{0} & \mathbf{A}_{\boldsymbol{\theta}_{0} \boldsymbol{\theta}_{0}}^{*}
\end{array}\right), \mathbf{B}_{\underline{\boldsymbol{\theta}}_{0}} \triangleq\left(\begin{array}{cc}
\mathbf{J}_{\boldsymbol{\theta}_{0}} & \mathbf{P}_{\boldsymbol{\theta}_{0}} \\
\mathbf{P}_{\boldsymbol{\theta}_{0}}^{*} & \mathbf{J}_{\boldsymbol{\theta}_{0}}^{*}
\end{array}\right),
$$

where

$$
\mathbf{A}_{\boldsymbol{\theta}_{0} \boldsymbol{\theta}_{0}}=E_{p}\left\{\nabla_{\boldsymbol{\theta}_{0}}^{H} \nabla_{\boldsymbol{\theta}_{0}} \ln f_{Z}\left(\mathbf{z} ; \underline{\boldsymbol{\theta}}_{0}\right)\right\},
$$

$$
\begin{gathered}
\mathbf{A}_{\boldsymbol{\theta}_{0}^{*} \boldsymbol{\theta}_{0}}=E_{p}\left\{\nabla_{\boldsymbol{\theta}_{0}}^{T} \nabla_{\boldsymbol{\theta}_{0}} \ln f_{Z}\left(\mathbf{z} ; \underline{\boldsymbol{\theta}}_{0}\right)\right\}, \\
\mathbf{J}_{\boldsymbol{\theta}_{0}}=E_{p}\left\{\nabla_{\boldsymbol{\theta}_{0}} \ln f_{Z}\left(\mathbf{z} ; \underline{\boldsymbol{\theta}}_{0}\right) \nabla_{\boldsymbol{\theta}_{0}}^{H} \ln f_{Z}\left(\mathbf{z} ; \underline{\boldsymbol{\theta}}_{0}\right)\right\}, \\
\mathbf{P}_{\boldsymbol{\theta}_{0}}=E_{p}\left\{\nabla_{\boldsymbol{\theta}_{0}} \ln f_{Z}\left(\mathbf{z} ; \underline{\boldsymbol{\theta}}_{0}\right) \nabla_{\boldsymbol{\theta}_{0}}^{T} \ln f_{Z}\left(\mathbf{z} ; \underline{\boldsymbol{\theta}}_{0}\right)\right\},
\end{gathered}
$$

where the complex gradient $\nabla_{\boldsymbol{\theta}_{0}}$ is defined as in (7). $\mathbf{J}_{\boldsymbol{\theta}_{0}}$ and $\mathbf{P}_{\boldsymbol{\theta}_{0}}$ are the Misspecified Information Matrix (MIM) and Misspecified Pseudo-Information Matrix (MPIM).

Proof: Using the relation in (12), the matrix $\mathbf{A}_{\tilde{\boldsymbol{\theta}}_{0}}$ can be rewritten as function of the complex augmented pseudo-true parameter vector $\underline{\theta}_{0}$ as:

$$
\mathbf{A}_{\tilde{\boldsymbol{\theta}}_{0}}=\mathbf{T}^{H} E_{p}\left\{\frac{\partial}{\partial \underline{\boldsymbol{\theta}}_{0}}\left(\frac{\partial \ln f_{Z}\left(\mathbf{z} ; \underline{\boldsymbol{\theta}}_{0}\right)}{\partial \underline{\boldsymbol{\theta}}_{0}}\right)^{H}\right\} \mathbf{T}=\mathbf{T}^{H} \mathbf{A}_{\underline{\boldsymbol{\theta}}_{0}} \mathbf{T}
$$

Similarly, (10) allows us to recast the matrix $\mathbf{B}_{\tilde{\theta}_{0}}$ as function of $\underline{\theta}_{0}$ :

$$
\begin{aligned}
\mathbf{B}_{\tilde{\boldsymbol{\theta}}_{0}} & \triangleq \mathbf{T}^{T} E_{p}\left\{\left(\frac{\partial \ln f_{Z}\left(\mathbf{z} ; \underline{\boldsymbol{\theta}}_{0}\right)}{\partial \underline{\boldsymbol{\theta}}_{0}}\right)^{T} \frac{\partial \ln f_{Z}\left(\mathbf{z} ; \underline{\boldsymbol{\theta}}_{0}\right)}{\partial \underline{\boldsymbol{\theta}}_{0}}\right\} \mathbf{T} \\
& =\mathbf{T}^{T} \mathbf{S B}_{\underline{\boldsymbol{\theta}}_{0}} \mathbf{T}=2 \mathbf{T}^{-1} \mathbf{B}_{\underline{\boldsymbol{\theta}}_{0}} \mathbf{T}=\mathbf{T}^{H} \mathbf{B}_{\underline{\boldsymbol{\theta}}_{0}} \mathbf{T},
\end{aligned}
$$

where $\mathbf{S}=\left(\begin{array}{ll}\mathbf{0} & \mathbf{I} \\ \mathbf{I} & \mathbf{0}\end{array}\right)$. Using the mapping in (3) and the definitions in (18) and (21), we have:

$$
\mathbf{C}\left(\tilde{\hat{\boldsymbol{\theta}}}, \tilde{\boldsymbol{\theta}}_{0}\right)=4^{-1} \mathbf{T}^{H} \mathbf{C}\left(\underline{\hat{\boldsymbol{\theta}}}, \underline{\boldsymbol{\theta}}_{0}\right) \mathbf{T} .
$$

By substituting (29), (30) and (31) in (17), and by relying on the properties of $\mathbf{T}$, we get:

$$
\begin{gathered}
\mathbf{T}^{H} \mathbf{C}\left(\underline{\hat{\theta}}, \underline{\boldsymbol{\theta}}_{0}\right) \mathbf{T} \geqslant 4\left[\mathbf{T}^{H} \mathbf{A}_{\underline{\boldsymbol{\theta}}_{0}} \mathbf{T}\right]^{-1} \mathbf{T}^{H} \mathbf{B}_{\underline{\theta}_{0}} \mathbf{T} \times \\
\times\left[\mathbf{T}^{H} \mathbf{A}_{\underline{\boldsymbol{\theta}}_{0}} \mathbf{T}\right]^{-1}=\mathbf{T}^{H} \mathbf{A}_{\underline{\theta}_{0}}^{-1} \mathbf{B}_{\underline{\theta}_{0}} \mathbf{A}_{\underline{\theta}_{0}}^{-1} \mathbf{T} .
\end{gathered}
$$

Finally, we can observe that, from (32):

$$
\mathbf{T}^{H}\left[\mathbf{C}\left(\underline{\hat{\boldsymbol{\theta}}}, \underline{\boldsymbol{\theta}}_{0}\right)-\mathbf{A}_{\underline{\boldsymbol{\theta}}_{0}}^{-1} \mathbf{B}_{\underline{\boldsymbol{\theta}}_{0}} \mathbf{A}_{\underline{\boldsymbol{\theta}}_{0}}^{-1}\right] \mathbf{T} \geq \mathbf{0},
$$

then, from the properties of the unitary matrices, we obtain the inequality in (20). This concludes the proof.

It is easy to verify that, when there is no mismatch, i.e. when $\mathbf{B}_{\underline{\theta}_{0}}=-\mathbf{A}_{\underline{\theta}_{0}}$, the bound in (20) is equal to the one derived in [6] and [9] for unbiased complex estimators.

Corollary 1. If the matrix $\mathbf{A}_{\boldsymbol{\theta}_{0}^{*} \boldsymbol{\theta}_{0}}$ in (26) vanishes, then:

$$
\mathbf{C}_{\mathbf{C}}\left(\hat{\boldsymbol{\theta}}, \boldsymbol{\theta}_{0}\right) \geq \mathbf{A}_{\boldsymbol{\theta}_{0} \boldsymbol{\theta}_{0}}^{-1} \mathbf{J}_{\boldsymbol{\theta}_{0}} \mathbf{A}_{\boldsymbol{\theta}_{0}}^{-1} \boldsymbol{\theta}_{0} .
$$

Proof: The proof follows directly from the inequality (20) and from the properties of the principal sub-matrices of a positive semidefinite matrix (see e.g. [21]).

Even though Corollary 1 provides a bound on the error covariance matrix, it must be noted that, even if an MS-unbiased estimator attains the bound in (34), this does not imply that $\hat{\boldsymbol{\theta}}$ attains the MCRB in (20). In fact, the covariance matrix alone does not provide a complete second-order characterization of a complex random vector, and in particular, of a complex estimator. 


\section{THE COMPLEX CONSTRAINED MCRB}

This section provides a generalization to complex parameters of the CMCRB derived in [3]. Suppose that the complex mismatched estimator $\hat{\boldsymbol{\theta}}$ is required to satisfy $k$ (with $k<d$ ) complex constraints $\mathbb{C}^{k}$ э $\mathbf{f}(\hat{\boldsymbol{\theta}})=\boldsymbol{\xi}(\hat{\boldsymbol{\theta}})+j \boldsymbol{\gamma}(\hat{\boldsymbol{\theta}})=\mathbf{0}$. We assume that A1, A2 and A4, discussed in Sect. III hold true, while A3 needs to be modified as follows:

A5 The function $E_{p}\left\{\ln f_{Z}(\mathbf{z} ; \boldsymbol{\theta}) \mid \boldsymbol{\theta} \in \bar{\Theta}\right\}$ has a unique maximum on $\bar{\Theta}=\left\{\boldsymbol{\theta} \in \mathbb{C}^{d} \mid \mathbf{f}(\boldsymbol{\theta})=\mathbf{0}\right\}$ in an interior point $\boldsymbol{\theta}_{0}$.

As before, $\boldsymbol{\theta}_{0}$ is the constrained pseudo-true parameter vector, i.e. the point that minimizes the KL divergence between $p_{Z}(\mathbf{z})$ and $f_{Z}(\mathbf{z} ; \boldsymbol{\theta})$. For an exhaustive discussion on the existence and on the properties of the real representation $\tilde{\boldsymbol{\theta}}_{0}$ of the constrained pseudo-true parameter vector, we refer the reader to [3].

As for the unconstrained case, to derive the complex CMCRB, we rely on the proof of its real form given in [3]. We define the $2 k \times 2 d$ real Jacobian matrix of the real representation of the constraints $\tilde{\mathbf{f}}(\tilde{\boldsymbol{\theta}})=\left[\begin{array}{ll}\boldsymbol{\xi}(\tilde{\boldsymbol{\theta}})^{T} & \gamma(\tilde{\boldsymbol{\theta}})^{T}\end{array}\right]^{T}$ as $\mathbf{F}_{\tilde{\boldsymbol{\theta}}} \triangleq \partial \tilde{\mathbf{f}}(\tilde{\boldsymbol{\theta}}) / \partial \tilde{\boldsymbol{\theta}} \in \mathbb{R}^{2 k \times 2 d}$, where $\partial \tilde{\mathbf{f}}(\tilde{\boldsymbol{\theta}}) / \partial \tilde{\boldsymbol{\theta}}$ represents the extension of the cogradient operator in (8) to vector valued functions:

$$
\partial \tilde{\mathbf{f}} / \partial \tilde{\boldsymbol{\theta}} \triangleq\left[\begin{array}{lll}
\left(\partial \tilde{f}_{1} / \partial \tilde{\boldsymbol{\theta}}\right)^{T} & \cdots & \left(\partial \tilde{f}_{2 k} / \partial \tilde{\boldsymbol{\theta}}\right)^{T}
\end{array}\right]^{T}
$$

We assume that $\operatorname{rank}\left(\mathbf{F}_{\tilde{\boldsymbol{\theta}}}\right)=2 k$, i.e. the constraints are non redundant and $\mathbf{F}_{\tilde{\boldsymbol{\theta}}}$ has full row rank. Then there exists $\mathbf{U} \in \mathbb{R}^{2 d \times(2 d-2 k)}$ whose columns form an orthonormal basis for the null space of $\mathbf{F}_{\tilde{\boldsymbol{\theta}}}$, i.e. $\mathbf{F}_{\tilde{\boldsymbol{\theta}}} \mathbf{U}=\mathbf{0}$ and $\mathbf{U}^{T} \mathbf{U}=\mathbf{I}$. At this point, Theorem 2 in [3] can be recast as:

Lemma 2. Let $\tilde{\hat{\theta}}$ be the real representation of a constrained MS-unbiased estimator $\hat{\boldsymbol{\theta}} \in \bar{\Theta}$ derived under the misspecified model $\mathcal{F}$ from the data vector $\mathbf{z}$. Then, if $\mathbf{U}^{T} \mathbf{A}_{\tilde{\boldsymbol{\theta}}_{0}} \mathbf{U}$ is nonsingular, for every $p_{Z}(\tilde{\mathbf{z}})$ in $\mathcal{P}$ :

$$
\begin{aligned}
\mathbf{C}\left(\tilde{\hat{\boldsymbol{\theta}}}, \tilde{\boldsymbol{\theta}}_{0}\right) & \geqslant \mathbf{U}\left(\mathbf{U}^{T} \mathbf{A}_{\tilde{\boldsymbol{\theta}}_{0}} \mathbf{U}\right)^{-1} \mathbf{U}^{T} \mathbf{B}_{\tilde{\boldsymbol{\theta}}_{0}} \mathbf{U} \times \\
& \times\left(\mathbf{U}^{T} \mathbf{A}_{\tilde{\boldsymbol{\theta}}_{0}} \mathbf{U}\right)^{-1} \mathbf{U}^{T} \triangleq \operatorname{CMCRB}\left(\tilde{\boldsymbol{\theta}}_{0}\right),
\end{aligned}
$$

where $\mathbf{C}\left(\tilde{\hat{\boldsymbol{\theta}}}, \tilde{\boldsymbol{\theta}}_{0}\right), \mathbf{A}_{\tilde{\boldsymbol{\theta}}_{0}}$ and $\mathbf{B}_{\tilde{\boldsymbol{\theta}}_{0}}$ are defined in (18), (15) and (19), respectively.

Proof: The proof follows directly from the results in [3].

Exactly as for Theorem 1, we show that, by using the mappings in (1), (2) and (3), it is possible to establish the CMCRB directly in the complex field. We start by defining the $2 k \times 2 d$ Jacobian matrix of the complex augmented representation of the constraints $\underline{\mathbf{f}}(\underline{\boldsymbol{\theta}})=\left[\begin{array}{ll}\mathbf{f}(\underline{\boldsymbol{\theta}})^{T} & \mathbf{f}(\underline{\boldsymbol{\theta}})^{H}\end{array}\right]^{T}$ as $\mathbf{F}_{\underline{\boldsymbol{\theta}}} \triangleq \partial \underline{\mathbf{f}}(\underline{\boldsymbol{\theta}}) / \partial \underline{\boldsymbol{\theta}} \in \mathbb{C}^{2 k \times 2 d}$, where $\partial \underline{\mathbf{f}}(\underline{\boldsymbol{\theta}}) / \partial \underline{\boldsymbol{\theta}}$ represents the extension of the cogradient operator in eq. (9) to vector valued functions:

$$
\partial \underline{\mathbf{f}} / \partial \underline{\boldsymbol{\theta}} \triangleq\left[\begin{array}{lll}
\left(\partial \underline{f}_{1} / \partial \underline{\boldsymbol{\theta}}\right)^{T} & \cdots & \left(\partial \underline{f}_{2 k} / \partial \underline{\boldsymbol{\theta}}\right)^{T}
\end{array}\right]^{T} .
$$

It is easy to verify through direct calculation (see also [7]) that:

$$
\mathbf{F}_{\tilde{\boldsymbol{\theta}}}=2^{-1} \mathbf{T}_{2 k}^{H} \mathbf{F}_{\underline{\boldsymbol{\theta}}} \mathbf{T}_{2 d}
$$

where the subscripts $2 d$ and $2 k$ indicates the size of the matrix $\mathbf{T}$. Then $2 k=\operatorname{rank}\left(\mathbf{F}_{\tilde{\boldsymbol{\theta}}}\right)=\operatorname{rank}\left(\mathbf{F}_{\boldsymbol{\theta}}\right)$, i.e. $\mathbf{F}_{\boldsymbol{\theta}}$ has full row rank. Consequently, there exists $\boldsymbol{\Delta} \in \mathbb{C}^{2 d \times \overline{(2} d-2 k)}$ whose columns form an orthonormal basis for the null space of $\mathbf{F}_{\underline{\theta}}$, i.e. $\mathbf{F}_{\boldsymbol{\theta}} \boldsymbol{\Delta}=\mathbf{0}$ and $\boldsymbol{\Delta}^{H} \boldsymbol{\Delta}=\mathbf{I}$ ( [7], [9]). The following theorem provide the complex form of the CMCRB.

Theorem 2. Let $\underline{\hat{\boldsymbol{\theta}}}$ be the augmented complex representation of a constrained $M S$-unbiased estimator $\hat{\boldsymbol{\theta}} \in \bar{\Theta}$ derived under the misspecified model $\mathcal{F}$ from the data vector $\mathbf{z}$. Then, if $\boldsymbol{\Delta}^{H} \mathbf{A}_{\underline{\boldsymbol{\theta}}_{0}} \boldsymbol{\Delta}$ is non-singular, for every $p_{Z}(\mathbf{z})$ in $\mathcal{P}$ :

$$
\begin{aligned}
\mathbf{C}\left(\underline{\hat{\boldsymbol{\theta}}}, \underline{\boldsymbol{\theta}}_{0}\right) & \geqslant \boldsymbol{\Delta}\left(\boldsymbol{\Delta}^{H} \mathbf{A}_{\underline{\boldsymbol{\theta}}_{0}} \boldsymbol{\Delta}\right)^{-1} \boldsymbol{\Delta}^{H} \mathbf{B}_{\underline{\boldsymbol{\theta}}_{0}} \boldsymbol{\Delta} \times \\
& \times\left(\boldsymbol{\Delta}^{H} \mathbf{A}_{\underline{\boldsymbol{\theta}}_{0}} \boldsymbol{\Delta}\right)^{-1} \boldsymbol{\Delta}^{H} \triangleq \operatorname{CMCRB}\left(\underline{\boldsymbol{\theta}}_{0}\right),
\end{aligned}
$$

where $\mathbf{C}\left(\underline{\hat{\boldsymbol{\theta}}}, \underline{\boldsymbol{\theta}}_{0}\right), \mathbf{A}_{\underline{\theta}_{0}}$ and $\mathbf{B}_{\underline{\theta}_{0}}$ are defined in (21) and (24), respectively.

Proof: As in Theorem 1, we can transform $\mathbf{C}\left(\tilde{\hat{\boldsymbol{\theta}}}, \tilde{\boldsymbol{\theta}}_{0}\right), \mathbf{A}_{\tilde{\boldsymbol{\theta}}_{0}}$ and $\mathbf{B}_{\tilde{\boldsymbol{\theta}}_{0}}$ by using the relations in (31), (29) and (30), respectively. Moreover, by defining $\mathbf{D}=\mathbf{T}_{2 d} \mathbf{U}$ and by noticing that, since $\mathbf{U}$ is a real matrix, $\mathbf{U}^{T}=\mathbf{U}^{H}$, from (36) we get:

$$
\begin{array}{r}
\mathbf{T}_{2 d}^{H} \mathbf{C}\left(\underline{\hat{\boldsymbol{\theta}}}, \underline{\boldsymbol{\theta}}_{0}\right) \mathbf{T}_{2 d} \geqslant \mathbf{T}_{2 d}^{H} \mathbf{D}\left(\mathbf{D}^{H} \mathbf{A}_{\underline{\boldsymbol{\theta}}_{0}} \mathbf{D}\right)^{-1} \times \\
\times \mathbf{D}^{H} \mathbf{B}_{\underline{\boldsymbol{\theta}}_{0}} \mathbf{D}\left(\mathbf{D}^{H} \mathbf{A}_{\underline{\boldsymbol{\theta}}_{0}} \mathbf{D}\right)^{-1} \mathbf{D}^{H} \mathbf{T}_{2 d} .
\end{array}
$$

From (38) and from the definition of the matrix $\mathbf{U}$, we have that $\mathbf{F}_{\tilde{\boldsymbol{\theta}}} \mathbf{U}=2^{-1} \mathbf{T}_{2 k}^{H} \mathbf{F}_{\underline{\boldsymbol{\theta}}} \mathbf{T}_{2 d} \mathbf{U}=\mathbf{0}$, then $\mathbf{F}_{\underline{\boldsymbol{\theta}}} \mathbf{T}_{2 d} \mathbf{U}=\mathbf{F}_{\underline{\boldsymbol{\theta}}} \mathbf{D}=$ $\mathbf{0}$. Then, since $\mathbf{F}_{\tilde{\boldsymbol{\theta}}}$ has full row rank, $\mathbf{D}$ and $\boldsymbol{\Delta}$ span the same subspace, then there exists an invertible $(2 d-2 k) \times(2 d-2 k)$ transformation $\mathbf{L}$ such that $\mathbf{D L}=\Delta$. Finally, the inequality in (40) can be rewritten as:

$$
\begin{aligned}
& \mathbf{C}\left(\underline{\hat{\boldsymbol{\theta}}}, \underline{\boldsymbol{\theta}}_{0}\right) \geqslant \boldsymbol{\Delta} \mathbf{L}^{-1}\left[\mathbf{L}^{-H} \boldsymbol{\Delta}^{H} \mathbf{A}_{\underline{\boldsymbol{\theta}}_{0}} \mathbf{D} \boldsymbol{\Delta} \mathbf{L}^{-1}\right]^{-1} \times \\
& \times \mathbf{L}^{-H} \boldsymbol{\Delta}^{H} \mathbf{B}_{\underline{\boldsymbol{\theta}}_{0}} \boldsymbol{\Delta} \mathbf{L}^{-1}\left[\mathbf{L}^{-H} \boldsymbol{\Delta}^{H} \mathbf{A}_{\underline{\boldsymbol{\theta}}_{0}} \mathbf{D} \boldsymbol{\Delta} \mathbf{L}^{-1}\right]^{-1} \mathbf{L}^{-H} \boldsymbol{\Delta}^{H} \\
& =\boldsymbol{\Delta}\left[\boldsymbol{\Delta}^{H} \mathbf{A}_{\underline{\boldsymbol{\theta}}_{0}} \boldsymbol{\Delta}\right]^{-1} \boldsymbol{\Delta}^{H} \mathbf{B}_{\underline{\boldsymbol{\theta}}_{0}} \boldsymbol{\Delta}\left[\boldsymbol{\Delta}^{H} \mathbf{A}_{\underline{\boldsymbol{\theta}}_{0}} \boldsymbol{\Delta}\right]^{-1} \boldsymbol{\Delta}^{H}
\end{aligned}
$$

This conclude the proof.

Also in this case, it is trivial to verify that, when there is no mismatch, i.e. when $\mathbf{B}_{\underline{\theta}_{0}}=-\mathbf{A}_{\underline{\theta}_{0}}$, the bound in (39) is equal to the one derived [7] and [9].

\section{AN EXAMPLE: MISSPECIFICATION OF THE CIRCULARITY}

Inspired by the example reported in [6], in this section, we show how to apply Theorem 1 to a simple mismatched Least Square problem. Suppose that the complex data vector $\mathbf{z} \in \mathbb{C}^{N}$ follows a linear measurement model:

$$
\mathbf{z}=\boldsymbol{X} \overline{\boldsymbol{\theta}}+\mathbf{n}
$$

where $\overline{\boldsymbol{\theta}} \in \mathbb{C}^{d}$ is the complex unknown parameter vector to be estimated, $\mathbf{X} \in \mathbb{C}^{N \times d}$ is a known full rank complex matrix and $\mathbf{n} \in \mathbb{C}^{N}$ is a zero-mean complex random vector. We analyse the following study case:

- the true but unknown or inaccessible pdf of $\mathbf{z}, p_{Z}(\mathbf{z})$, is a non-circular complex Gaussian distribution [15], 
- the assumed pdf $f_{Z}(\mathbf{z} ; \boldsymbol{\theta})$ used for data inference is a circular complex Gaussian distribution,

i.e. we misspecify the circularity property of the collected data vector. Specifically, the true pdf of $\mathbf{z}$ can be expressed as [15]:

$$
p_{Z}(\mathbf{z})=\pi^{-N}|\boldsymbol{\Gamma}|^{-1 / 2}|\boldsymbol{\Xi}|^{-1 / 2} \exp (q(\mathbf{a}) / 2)
$$

where:

$$
q(\mathbf{a})=\left(\begin{array}{ll}
\mathbf{a}^{H} & \mathbf{a}^{T}
\end{array}\right)\left(\begin{array}{cc}
\boldsymbol{\Gamma} & \boldsymbol{\Xi} \\
\boldsymbol{\Xi}^{*} & \boldsymbol{\Gamma}^{*}
\end{array}\right)\left(\begin{array}{l}
\mathbf{a} \\
\mathbf{a}^{*}
\end{array}\right),
$$

and $\mathbf{a} \triangleq \mathbf{z}-\mathbf{X} \overline{\boldsymbol{\theta}}$. According to the misspecified estimation framework ( [1], [2], [3]), we assume for $\mathbf{z}$ a misspecified circular Gaussian distribution:

$$
f_{Z}(\mathbf{z} ; \boldsymbol{\theta})=\pi^{-N}|\boldsymbol{\Gamma}|^{-1 / 2} \exp \left((\mathbf{z}-\mathbf{X} \boldsymbol{\theta})^{H} \boldsymbol{\Gamma}^{-1}(\mathbf{z}-\mathbf{X} \boldsymbol{\theta})\right) .
$$

For simplicity, following [6], we assume that $\boldsymbol{\Gamma}=\sigma^{2} \mathbf{I}_{N}, \boldsymbol{\Xi}=$ $\tau \mathbf{I}_{N}, \sigma^{2}>0$ and $\tau \in \mathbb{C}$.

Our aim here is to show how Theorem 1 can be applied to obtain the complex MCRB for the linear Least Squares estimation problem in (42). At first, we need to evaluate the pseudo-true parameter vector $\boldsymbol{\theta}_{0}$ defined in (16). Under suitable regularity conditions and under Assumptions A1-A4, $\boldsymbol{\theta}_{0}$ can be evaluated by solving the following equation:

$$
\left.\nabla_{\boldsymbol{\theta}} D\left(p_{Z} \| f_{\boldsymbol{\theta}}\right)\right|_{\boldsymbol{\theta}=\boldsymbol{\theta}_{0}}=\left.\nabla_{\boldsymbol{\theta}} E_{p}\left\{\ln f_{Z}(\mathbf{z} ; \boldsymbol{\theta})\right\}\right|_{\boldsymbol{\theta}=\boldsymbol{\theta}_{0}}=\mathbf{0} .
$$

Through direct calculation and neglecting the constant terms, by substituting (45) in (46) we have:

$$
\begin{gathered}
\left.\nabla_{\boldsymbol{\theta}} E_{p}\left\{(\mathbf{z}-\mathbf{X} \boldsymbol{\theta})^{H} \boldsymbol{\Gamma}^{-1}(\mathbf{z}-\mathbf{X} \boldsymbol{\theta})\right\}\right|_{\boldsymbol{\theta}=\boldsymbol{\theta}_{0}} \\
=\left.E_{p}\left\{\mathbf{X}^{H} \mathbf{z}-\mathbf{X}^{H} \mathbf{X} \boldsymbol{\theta}\right\}\right|_{\boldsymbol{\theta}=\boldsymbol{\theta}_{0}}=\mathbf{0},
\end{gathered}
$$

then $E_{p}\{\mathbf{z}\}=\mathbf{X} \boldsymbol{\theta}_{0}$ and then, from (42), $\boldsymbol{\theta}_{0}=\overline{\boldsymbol{\theta}}$. Now we pass to evaluate the matrices $\mathbf{A}_{\underline{\theta}_{0}}$ and $\mathbf{B}_{\underline{\theta}_{0}}$, i.e. the pseudotrue parameter vector is equal to the true one. At this point, through some algebra, it is immediate to verify that:

$$
\begin{gathered}
\mathbf{A}_{\underline{\boldsymbol{\theta}}_{0}}=\left(\begin{array}{cc}
\sigma^{-2} \mathbf{X}^{H} \mathbf{X} & \mathbf{0} \\
\mathbf{0} & \mathbf{X}^{T} \mathbf{X}^{*}
\end{array}\right), \\
\mathbf{B}_{\underline{\theta}_{0}}=\left(\begin{array}{cc}
\sigma^{-2} \mathbf{X}^{H} \mathbf{X} & \sigma^{-4} \tau \mathbf{X}^{H} \mathbf{X}^{*} \\
\sigma^{-4} \tau^{*} \mathbf{X}^{T} \mathbf{X} & \sigma^{-2} \mathbf{X}^{T} \mathbf{X}^{*}
\end{array}\right) .
\end{gathered}
$$

By collecting the previous results, we have that $\operatorname{MCRB}\left(\boldsymbol{\theta}_{0}\right) \equiv \operatorname{MCRB}(\overline{\boldsymbol{\theta}})$ and:

$$
\operatorname{MCRB}(\overline{\boldsymbol{\theta}})=\left(\begin{array}{cc}
\sigma^{2}\left(\mathbf{X}^{H} \mathbf{X}\right)^{-1} & \tau\left(\mathbf{X}^{T} \mathbf{X}\right)^{-1} \\
\tau^{*}\left(\mathbf{X}^{T} \mathbf{X}\right)^{-*} & \sigma^{2}\left(\mathbf{X}^{H} \mathbf{X}\right)^{-*}
\end{array}\right) .
$$

When $\tau=0, p_{Z}(\mathbf{z})$ collapses to a circular Gaussian distribution, then there exists a vector $\overline{\boldsymbol{\theta}} \in \bar{\Theta}$ such that $p_{Z}(\mathbf{z})=f_{Z}(\mathbf{z} ; \overline{\boldsymbol{\theta}})$ i.e. the model is correctly specified. Consequently, the $\operatorname{MCRB}(\overline{\boldsymbol{\theta}})$ reduces to the classical CRB under circularity assumption for the linear model in (42), i.e. $\operatorname{CRB}(\overline{\boldsymbol{\theta}})=\sigma^{2}\left(\mathbf{X}^{H} \mathbf{X}\right)^{-1}[6]$.

\section{CONCLUSION}

In this paper, the complex form of the MCRB (Theorem 1) and of the Constrained MCRB (Theorem 2) is provided. Future works will investigate the efficiency of a given complex estimator with respect to the derived bounds.

\section{ACKNOWLEDGEMENT}

This material is based upon work partially supported by the Air Force Office of Scientific Research under award number FA9550-17-1-0065.

\section{REFERENCES}

[1] C. D. Richmond, L. L. Horowitz, "Parameter Bounds on Estimation Accuracy Under Model Misspecification," IEEE Trans. Signal Process., vol. 63, no. 9, pp. 2263-2278, May 1, 2015.

[2] S. Fortunati, F. Gini, M. S. Greco, "The Misspecified Cramér-Bound and its Application to the Scatter Matrix estimation in Complex Elliptically Symmetric distributions," IEEE Trans. Signal Process., vol. 64, no. 9, pp. 2387-2399, May 2016.

[3] S. Fortunati, F. Gini, M. S. Greco, 'The Constrained Misspecified CramérBound," IEEE Signal Process. Lett., vol.23, no.5, pp.718-721, May 2016.

[4] Q. H. Vuong, "Cramér-Rao bounds for misspecified models," Working paper 652, Division of the Humanities and Social Sciences, Caltech, October. 1986 (https://www.hss.caltech.edu/content/cramer-rao-boundsmisspecified-models).

[5] C. Ren, M. N. El Korso, J. Galy, E. Chaumette, P. Larzabal, A. Renaux, "Performances bounds under misspecification model for MIMO Radar application", EUSIPCO 2015, Nice, France.

[6] E. Ollila, V. Koivunen and J. Eriksson, "On the Cramér-Rao bound for the constrained and unconstrained complex parameters," 20085 th IEEE Sensor Array and Multichannel Signal Processing Workshop, SAM, Darmstadt, 2008, pp. 414-418.

[7] A. K. Jagannatham and B. D. Rao, "Cramér-Rao lower bound for constrained complex parameters," IEEE Signal Process. Lett., vol. 11, no. 11 , pp. $875-878$, Nov. 2004.

[8] A. van den Bos, "A Cramér-Rao lower bound for complex parameters," IEEE Trans. Signal Process., vol. 42, no. 10, pp. 2859-, Oct 1994.

[9] T. Menni, E. Chaumette, P. Larzabal and J. P. Barbot, "New Results on Deterministic CramérRao Bounds for Real and Complex Parameters," IEEE Trans. Signal Process., vol. 60, no. 3, pp. 1032-1049, March 2012.

[10] P. J. Schreirer and L. L. Scharf, Statistical Signal Processing of ComplexValued Data: the Theory of Improper and Noncircular Signals, Cambridge UK: Cambridge Univ. Press, 2010.

[11] T. Adali, P. J. Schreier and L. L. Scharf, "Complex-Valued Signal Processing: The Proper Way to Deal With Impropriety," IEEE Trans. Signal Process., vol. 59, no. 11, pp. 5101-5125, Nov. 2011.

[12] J. Eriksson, E. Ollila and V. Koivunen, "Essential Statistics and Tools for Complex Random Variables," IEEE Trans. Signal Process., vol. 58, no. 10 , pp. 5400-5408, Oct. 2010.

[13] S. C. Olhede, "On probability density functions for complex variables," IEEE Trans. Inf. Theory, vol. 52, no. 3, pp. 1212-1217, March 2006.

[14] B. Picinbono, "On circularity," IEEE Trans. Signal Process., vol. 42, no. 12, pp. 3473-3482, Dec 1994.

[15] B. Picinbono, "Second-order complex random vectors and normal distributions," IEEE Trans. Signal Process., vol. 44, no. 10, pp. 2637-2640, Oct 1996.

[16] R. Remmert, Theory of Complex Functions. New York: Springer, 1991.

[17] K. Kreutz-Delgado, 'The complex gradient operator and the CRcalculus," ArXiv:0906.4835v1, [math.OC], 2009.

[18] D. H. Brandwood, "A complex gradient operator and its application in adaptive array theory," Proc. IEEE - Communications, Radar and Signal Processing, vol. 130, no. 1, pp. 11-16, February 1983.

[19] A. van den Bos, "Complex gradient and Hessian," Proc. IEE - Vision, Image and Signal Processing, vol. 141, no. 6, pp. 380-383, Dec 1994.

[20] H. Li and T. Adali, Complex-Valued Adaptive Signal Processing Using Nonlinear Functions, EURASIP Journal on Advances in Signal Processing, 2008, 2008:765615.

[21] R. A. Horn, C. A. Johnson, Matrix Analysis, Cambridge University Press, New York, 1985. 